\title{
Deportación y violación de los derechos del migrante en ambas fronteras
}

\author{
Deportation and violation of the rights of migrants on both borders
}

\author{
Martha Guerrero-Ortiz / marthaguerrero_5@hotmail.com \\ Universidad Autónoma de Zacatecas, México \\ Martha Cecilia Jaramillo-Cardona / martha.jaramillo@uabc.edu.mx \\ Universidad Autónoma de Baja California, México
}

\begin{abstract}
Many national and international organizations consider that the U.S. economic crisis is the reason behind a significant decrease of migratory flows, from and through Mexico, towards the United States in 2009. This has also increased the number of cases of abuse and deportation of migrants. This paper analyzes the cause for deportation of migrants from all over the United States to Mexico. Furthermore, we will depict the violence and abuse that this vulnerable group of people are subject to in Mexico, not only by municipal, federal or migration authorities, but by other actors of the migratory process, called "coyotes", who also violate migrant's human rights.
\end{abstract}

Key words: deportation, migration, United States, abuse, human rights.

Resumen: Diversos organismos internacionales y nacionales consideran la crisis económica estadounidense como la que ha provocado una caída significativa en los flujos migratorios desde y a través de México hacia Estados Unidos durante 2009, al mismo tiempo que también se han incrementado de forma considerable no sólo las deportaciones de los inmigrantes, sino el maltrato de que son objeto. En este sentido, el trabajo aquí presente analiza los motivos que originaron la deportación de los migrantes procedentes de los distintos lugares de Estados Unidos hacia México, y la forma en que éstos son maltratados y violentados dentro del territorio mexicano, y no únicamente por parte de las autoridades municipales, federales y de migración, sino por los diferentes actores que intervienen en estos procesos migratorios como los llamados "coyotes" que terminan vulnerando los derechos humanos de los migrantes.

Palabras clave: deportación, migración, Estados Unidos, maltrato, derechos humanos. 


\section{Introducción}

En el presente siglo la migración se enfatiza como uno de los temas mundiales determinantes, debido al incremento de traslados de personas de un lugar a otro. Esto se refleja en las estadísticas actuales donde cerca de 214 millones de personas viven fuera de su país de origen, lo cual representa alrededor de 3\% de la población mundial (Gómez, 2010: 294). También, de acuerdo con la Primera Cumbre de Comunidades Migrantes Latinoamericanas realizada en México en 2007, se consideró que existen cerca de 30 millones de migrantes latinoamericanos y caribeños fuera de sus países (Bassegio, 2007).

La migración, como consecuencia de las condiciones desiguales en el desarrollo económico de los países, continúa siendo un fenómeno constante en el mundo; sin embargo, pese a los grandes esfuerzos por regular el flujo migratorio, los resultados no han sido satisfactorios, los controles han sido extremos e incluso inhumanos y crueles. Basta recordar las condiciones de hacinamiento que prevalecen en las estaciones migratorias o en los traslados previos a las deportaciones de que son sujetos los migrantes indocumentados en ambas fronteras del territorio mexicano; o los migrantes marroquíes, ecuatorianos y colombianos, que se desplazan a territorio español, por citar algunos ejemplos. De ahí la importancia, como lo plantea (Martínez, 2003), de mostrar que los migrantes no son ni masas de desheredados ni amenazas para la seguridad nacional de ningún país.

Según cifras del Instituto Nacional de Migración (INM) en 2007, fueron sancionados 187 servidores públicos de dicha institución, principalmente por violar los derechos humanos de los migrantes a través del maltrato físico, la falta de alimentación y el mal servicio de las estaciones migratorias. A esta situación se le puede sumar también la persistencia de prácticas frecuentes de corrupción, autoritarismo y abuso por parte de algunas autoridades policiales consulares y migratorias, así como los peligros y riesgos propios de un largo viaje en medios no apropiados, los abusos y crímenes sufridos por la delincuencia organizada, nacional y extranjera, y las vejaciones discriminatorias de las que son víctimas los migrantes centroamericanos una vez que llegan a territorio mexicano (Fuentes y Ortiz, 2012).

Los migrantes indocumentados mexicanos y centroamericanos son vulnerables a los abusos no sólo de los empresarios estadounidenses, sino también de quienes lucran con este fenómeno. Quienes deciden migrar, inician un calvario desde que emprenden su partida en el lugar de origen, hasta llegar a las fronteras: sur de México para los centroamericanos, y norte para mexicanos y centroamericanos. En estos lugares es donde se agudiza más el 
sufrimiento debido a los múltiples problemas que deben enfrentar, siendo uno de ellos la relación a establecer con "el pollero" o "coyote". En ésta surge una serie de situaciones de riesgo por los prejuicios de quienes se dedican a esta clase de negocio, ya que el migrante es visto como mercancía, y los abusos de los negociadores pueden llegar a terminar en secuestro, robo, violaciones a las mujeres y muchas veces hasta la muerte de quienes por diversas circunstancias son abandonados en la travesía. Estos maltratos no diferencian si son hombres, mujeres o niños (Morales, 2008: 138).

Por años, los indocumentados han sido y siguen siendo blanco de políticas de control y deportación en Estados Unidos, políticas que también han ido acompañadas de la violación de los derechos humanos de los migrantes tanto por autoridades norteamericanas como mexicanas una vez deportados al territorio. Ante estos hechos es importante seguir investigando las causas y los motivos por los cuales muchos migrantes son deportados, y al mismo tiempo conocer la forma en que son tratados durante el proceso por las diversas autoridades.

En este sentido, algunos de los programas para contrarrestar la migración es el de "prevención por medio de la disuasión", iniciado en el año 1993 con Bill Clinton, debido a las altas tasas de migrantes indocumentados procedentes de México a causa de la crisis económica. Este programa de vigilancia a la frontera con México ya no se orienta a la detención y deportación de indocumentados, sino más bien buscó disuadir a quienes deseaban hacerlo (Cornelius, 2001).

Dentro de la estrategia sobresalen tres operaciones con la finalidad de evitar el cruce: la operación Bloqueo o Blockade (que pasó a llamarse Hold the Line), en Texas (El Paso, 1993); la Operación Guardián o Gatekeeper, en California (San Diego, 1994); y finalmente, la Operación Salvaguarda o Safeguard en Arizona (Tucson, 1995). En 2001 dicha situación se vio agravada tras los atentados del 11 de septiembre, momento a partir del cual se aplicaron medidas de contención más severas, que han ido acompañadas de un proceso constante de criminalización del migrante. Con la "Patriot Act" (Ley Patriota) cuyo propósito es "disuadir y castigar los actos terroristas en los Estados Unidos y alrededor del mundo, para mejorar las herramientas de investigación encargados de hacer cumplir la ley, y otros fines", la inmigración

1 Es el nombre que se le da a las personas que ayudan a los migrantes ilegales a cruzar la frontera entre México y Estados Unidos. Esta red trabaja en el cruce de quienes toman esta decisión con el cobro de cuotas específicas, acordadas entre los mismos inmigrantes y sus familiares (Morales, 2008:138). 
pasó a ser una cuestión de seguridad nacional, y poco después se convirtió en tendencia mundial (Perelló, 2013a: 58).

El programa Comunidades Seguras impulsado por Obama durante 2008 dio como resultado cifras récord de personas deportadas en su aplicación: 396,906 durante el año 2011 y 409,849 en 2012. Estas deportaciones masivas de migrantes, mayoritariamente de origen mexicano, sólo han buscado criminalizarlos. Sin embargo, aunque se pretende perseguir los crímenes más graves: asesinatos, violaciones, narcotráfico, etc., bajo el pretexto de mantener la seguridad, se está originando la deportación de personas que habían cometido delitos menores, simples faltas, o que incluso, en el peor de los casos, nunca habían delinquido, lo cual está dando lugar a numerosos casos de separación familiar (Perelló, 2013b: 69).

En este contexto, y con el fin de ahondar más en las causas que generan las deportaciones de mexicanos, el presente trabajo plantea las siguientes preguntas de investigación: ¿Cuáles son los motivos más frecuentes que originan la deportación y encarcelamiento de los migrantes mexicanos por región migratoria en ambos lados de la frontera norte? y ¿a qué tipo de violencia son sometidos los migrantes por las autoridades americanas y mexicanas?

En dicho sentido, el fin último de este trabajo es conocer y analizar los motivos que originaron la deportación y el tipo de violencia a los que son sometidos los migrantes tanto por autoridades americanas como mexicanas en el periodo 2006 y 2007. El análisis se realiza con base en la información recopilada a través de encuestas y con testimonios de los migrantes.

\section{Métodos y procedimientos}

Para este artículo se empleó información procedente de una encuesta aplicada en siete albergues para migrantes de Tijuana y Mexicali. La encuesta fue realizada por la organización Médicos del Mundo entre el 1 de enero de 2006 y el 31 de diciembre de 2007, donde los migrantes repatriados, deportados, con salida voluntaria de Estados Unidos, en tránsito, transmigrante o con intento de cruce a Estados Unidos sin éxito, acudieron para recibir de forma voluntaria atención médica.

La organización Médicos del Mundo ofrece servicios de salud gratuitos a la población vulnerable a nivel internacional, en el marco de un proyecto que se puso en marcha durante tres años en la frontera norte de México. Dicha institución estuvo vinculada con siete albergues de migrantes con el fin de ofrecer servicios en el área de salud, y al mismo tiempo obtener información de forma voluntaria de la situación migratoria de las personas atendidas. 
En este proyecto fueron atendidos 6,234 migrantes en consulta médica; de ellos sólo participaron en la encuesta $20.3 \%$, lo cual significa que en total se aplicaron 1,264 cuestionarios. Esto obedece a que las personas accedieron de forma voluntaria a responder la encuesta una vez recibida la atención médica, y por esta razón la muestra seleccionada para hacer el análisis y ofrecer los resultados que se presentan en este artículo es no aleatoria. En un gran porcentaje las preguntas que se incluyeron en esta encuesta eran cerradas, con opciones de respuesta múltiples, y algunas otras abiertas (el instrumento aplicado puede ser consultado en la versión electrónica del artículo en convergencia.uaemex.mx). Es importante señalar que las personas que accedieron a participar en la encuesta lo hicieron de manera informada y voluntaria.

El cuestionario se dividió en cuatro partes: 1) información general, 2) información acceso a la salud y tratamientos, 3) información migratoria y social, y 4) servicios de salud recibidos por los migrantes. Dada la amplia estructura del cuestionario, se tomaron para la elaboración de este artículo los datos relacionados con las causas por las que se dio la deportación a México y la forma en que fueron tratados por las autoridades migratorias y policiacas de Estados Unidos y México. Además de los datos estadísticos de la encuesta, se contó con los testimonios y relatos que algunos migrantes compartieron sobre su experiencia migratoria y se exponen como ejemplo, con el fin de ilustrar la forma en que son violados los derechos de la población migrante dentro de Estados Unidos y México.

La metodología empleada es mixta, se toman datos cuantitativos y cualitativos. Para los primeros se utilizó la base de datos en el Programa Estadístico para las Ciencias Sociales (SPSS, por sus siglas en inglés), misma con la que se procedió a hacer los cruces de las variables: los motivos de encarcelamiento que causó la deportación de Estados Unidos hacia la frontera de Tijuana y Mexicali, Baja California, México; su situación migratoria; nivel de escolaridad; tipo de violencia; autoridades culpables de ejercer violencia contra los migrantes en ambos lados de la frontera en relación con las cuatro regiones migratorias: la norte, la tradicional, la centro y la sur-sureste de México. De ello se obtienen las tablas de asociación de $\mathrm{r}$ x c, es decir, tablas de 4 por 4, de 5 por 4, de 6 por 4 de los datos categóricos (Cortés y Rubalcava, 1987: 105) que se muestran en el cuadro resumen del Anexo 1.

Para los datos cualitativos se tuvo en cuenta los testimonios que algunos migrantes expusieron voluntariamente en el momento de ser aplicado el cuestionario; de los diez testimonios se eligieron cuatro que ilustran la forma en que la población migrante es tratada en los dos países por donde realizan su travesía, México y Estados Unidos. 


\section{Motivos que originaron la deportación y encarcelamiento de los migrantes}

El hecho de cruzar una frontera encierra una serie de aspectos que llevan a una explicación causal de los movimientos poblacionales, y más aún en las fronteras como escenarios reales de la violación a los derechos humanos. Los abusos y agresiones están tipificadas en las fronteras, la línea que divide a las naciones, de donde se tiene una identidad y se forma otra o se adquiere otra, como parte de un proceso de integración (Morales, 2008).

La versión oficial de las autoridades estadounidenses considera que las deportaciones de los inmigrantes mexicanos se asocian con la migración indocumentada. Sin embargo, con base en los datos provenientes de la encuesta aplicada por Médicos del Mundo entre el primero de enero de 2006 y el 31 de diciembre de 2007 en albergues para migrantes de Tijuana y Mexicali, sobre la situación de salud de los migrantes y su acceso a la salud, se puede observar, entre otros aspectos, el maltrato, abuso y violencia contra los migrantes.

Entre los motivos de encarcelamiento que causó la deportación se encuentran, en primer lugar, las infracciones de tránsito y/o acumulación de multas con 28\%; en segundo lugar está el consumo de drogas y/o alcohol con $23 \%$; en tercer lugar, otros delitos o crímenes con $18 \%$; el cuarto lugar lo ocupa la venta de drogas con $12 \%$, y otro porcentaje igual por ser indocumentados; en quinto lugar, violencia doméstica con 6.3\%; y en sexto lugar, portación de papeles falsos con cerca de $1 \%$. Para ilustrar uno de estos casos se expone el de María, trabajadora indocumentada de 32 años, originaria de Acámbaro, Guanajuato.

Pasé mucho tiempo en el trabajo de cuidar niños, de hecho fue lo último que yo hice, tanto, que el día que me sacaron de Estados Unidos eran como las 18:00 horas. Pasé por una calle donde estaba una camioneta de la migración, que siempre estaba por allí, tanto la veía que siempre saludaba a los migras que estuvieran de turno, y esta vez estaba un güero y este cabrón me llamó y me preguntó por mis papeles, me preguntó como enojado, yo le contesté que no los traía porque venía del trabajo, el cabrón me dijo que me subiera a la patrulla para que lo llevara a mi casa, para que él viera mis documentos, como a las cuatro cuadras lo vi muy decidido a llegar hasta mi casa y le dije que le había mentido, que en realidad no tenía documentos, entonces se enojó mucho, me empezó a gritar, a decirme: "Maldita india, eres una mierda, sólo están aquí para apestar este país". En fin, me dijo muchas cosas y a mí me dio miedo verlo así, todo rojo como loco.

Después que el migra me dijo de todo, aceleró mucho la camioneta y me llevó hasta la línea, ahí pegadito a la barda, se paró, se bajó y me abrió la puerta de la camioneta, me dijo que me bajara, cuando bajé sacó su pistola y me la puso en la frente y me dijo: 
“Corre o te lleva la mierda, lárgate a México", y claro que corrí y brinqué la barda para este lado [México]. Ya de este lado estaba toda nerviosa porque me dio mucho miedo y escuché un disparo, no sé si me disparó a mí o al aire o eran mis nervios, pero seguí corriendo hasta llegar al centro de Tijuana.

Como este caso, son muchos los abusos que a diario viven los migrantes deportados de Estados Unidos a la frontera norte de México; independientemente de la causa de su deportación, el uso de la fuerza, el maltrato físico y verbal está presente. En este sentido, como lo plantean las Naciones Unidas y la CEPAL (2006), la migración internacional ofrece salidas al desempleo y a la falta de perspectivas de progreso laboral, pero a la vez genera crecientes riesgos para los migrantes, acentuando cada vez más su inseguridad, reflejada en la vulneración de los derechos humanos de muchos migrantes, ya sea a lo largo de su travesía, en el proceso de inserción en la sociedad de destino o durante la repatriación, golpeando especialmente a mujeres y niños que terminan siendo víctimas de la trata de personas (Gómez, 2010).

$\mathrm{Al}$ analizar los motivos de encarcelamiento y deportación migratoria por región, en primer lugar se puede ver que aparecen nuevamente las infracciones de tránsito. En estos casos los migrantes son originarios de las regiones centro de México, como los estados de Hidalgo, Morelos, Puebla, Querétaro, México, Tlaxcala y el Distrito Federal, y de sur-sureste, entre ellos los estados de Campeche, Chiapas, Guerrero, Oaxaca, Quintana Roo, Tabasco, Veracruz y Yucatán.

Los capturados por el consumo de drogas y/o alcohol son originarios de Jalisco, Guanajuato, Michoacán, Zacatecas, Aguascalientes, San Luis Potosí, Colima y Nayarit, y nuevamente la región del centro.

Respecto a la venta de drogas se ubica a los migrantes de la región norte de México como Baja California, Coahuila, Chihuahua, Nuevo León, Sonora, Tamaulipas, Sinaloa y Baja California Sur representada con $20 \%$ e incluso muy por arriba del promedio del país que es $12 \%$; y por violencia doméstica, con $8 \%$, a los migrantes de la región histórica o tradicional que corresponde a los estados de Jalisco, Guanajuato, Michoacán, Zacatecas, Aguascalientes, San Luis Potosí, Colima y Nayarit.

Con el afán de ilustrar lo antes señalado, se presenta como ejemplo el caso de Lorena, quien fue víctima de discriminación, violencia y maltrato cuando un agente de policía le ordenó que se detuviera mientras conducía para su casa. El agente de policía la azotó contra el vehículo que manejaba después de detenerla por una supuesta falta injustificada de tránsito, maltrato que recibió pese a estar embarazada. 
En relación con la situación migratoria que presentan los encarcelados, lo encabezan las personas que están en calidad de deportados con $40 \%$, seguido de los migrantes en tránsito con $30 \%$; en tercer lugar aparecen los repatriados con $26 \%$, y por último, con $1 \%$, lo representan aquellos migrantes que decidieron salir de Estados Unidos de forma voluntaria.

Estos mismos datos observados por regiones migratorias muestran que las deportaciones aparecen con igual o ligeramente superior porcentaje al nacional y a las regiones histórica y la del norte; enseguida, en el porcentaje de migrantes en tránsito aparecen los de las regiones del norte y la sur-sureste de México; los repatriados que son un poco más de una cuarta parte se sitúan por arriba de las regiones centro y sur-sureste, también ligeramente por debajo de este porcentaje la región histórica; y por último la salida voluntaria por parte de los migrantes originarios de las regiones centro y norte del país.

En cuanto al nivel escolar que presentan los migrantes que regresaron al país por cualquiera de las razones ya mencionadas, la mayoría cuenta con educación básica, aunque existe $5 \%$ de analfabetas. Por encima de este porcentaje se ubican las regiones histórica tradicional y la sur-sureste. En cambio para los migrantes con estudios universitarios se encontró cerca de $2 \%$, y ligeramente por arriba de este dato los migrantes de las regiones centro y norte del país. Es importante resaltar que el nivel de escolaridad de los migrantes también se convierte en un punto de referencia en el momento de conocer los casos, debido a que quienes poseen baja o nula escolaridad son más vulnerables para el abuso y maltrato por los traficantes de personas, las autoridades estadounidenses y mexicanas, por no conocer o poder leer sus derechos.

En este sentido, el derecho a la vida de los migrantes a veces corre peligro, en virtud, por ejemplo, de la aplicación coercitiva de prácticas de control de frontera e inmigración que pueden poner en peligro la vida y limitar o rehusar el acceso a la justicia en casos de violencia perpetrada por la policía o por funcionarios de servicios de fronteras o de inmigración, traficantes, empleadores, entre otros (Ceriani et al., 2015a).

El tema de los derechos de los migrantes es cuestionado continuamente, sobre todo cuando la situación de estas personas en cuanto a residencia o condición migratoria es indocumentada, y son objeto, sistemáticamente, de discriminación y violación de los derechos humanos. En el contexto del control de la migración los migrantes pueden ser objeto de verificaciones de identidad, privación de la libertad y deportación en condiciones que pueden infringir una serie de derechos civiles, culturales, económicos, políticos y sociales, hechos que van en contra de la igualdad y libertad ante la discriminación (Ceriani et al., 2015b). 


\section{La violencia contra los migrantes en los escenarios estadounidenses y en el país de origen}

Las estadísticas arrojadas por la encuesta señalan que la violencia sobre los migrantes en el escenario estadounidense es de la siguiente manera: 50\% de ellos sufre violencia física; $28 \%$, violencia verbal; $15 \%$, violencia física con robo; $12 \%$, robo de bienes; y $4 \%$, violencia psicológica.

Ante esta situación, cabe hacer las siguientes observaciones. Primero: la violencia física y verbal la sufren los migrantes originarios de la región histórica, es decir, los originarios de los estados de Jalisco, Guanajuato, Michoacán, Zacatecas, Aguascalientes, San Luis Potosí, Colima y Nayarit. Segundo: la violencia física y física con robo la sufren los migrantes de la región norte, es decir, Baja California, Coahuila, Chihuahua, Nuevo León, Sonora, Tamaulipas, Sinaloa y Baja California Sur. Tercero: la violencia física, verbal y física más robo se da contra los migrantes originarios de la región centro: Hidalgo, Morelos, Puebla, Querétaro, Distrito Federal, México y Tlaxcala, y la región sur-sureste: Campeche, Chiapas, Guerrero, Oaxaca, Quintana Roo, Tabasco, Veracruz y Yucatán.

Al hablar del tipo de violencia sobre los migrantes en el escenario mexicano, se puede ver que en primer lugar se produce la violencia física con robo, especialmente robo de bienes como dinero e identificaciones y la violencia física, verbal y psicológica. Respecto a la violencia física con robo, el promedio es de $27 \%$, sin embargo aparecen por arriba de este porcentaje las regiones, tradicional $^{2}$ y sur-sureste de México $^{3}$. El robo de bienes a los migrantes es de $24.3 \%$, donde por arriba de este porcentaje se sitúan tres de las cuatro regiones: la región tradicional, la región centro y la región sur-sureste de México, siendo la región norte ${ }^{4}$ la excepción. En cuanto a la violencia física el promedio es mayor $35.7 \%$, siendo la región norte la que aparece por arriba de este porcentaje, frente a las regiones tradicional y sur-sureste de México donde los datos aparecen más o menos similares al promedio. La violencia verbal que viven los migrantes es de $10 \%$ donde la región norte nuevamente está por arriba de este porcentaje. Y finalmente, con un promedio de $3.3 \%$ aparece la

2 Región que se conforma por los estados de Jalisco, Guanajuato, Michoacán, Zacatecas, Aguascalientes, San Luis Potosí, Colima y Nayarit.

3 La integran los estados de Campeche, Chiapas, Guerrero, Oaxaca, Quintana Roo, Tabasco, Veracruz y Yucatán.

4 Se conforma por los estados de Baja California, Coahuila, Chihuahua, Nuevo León, Sonora, Tamaulipas, Sinaloa y Baja California Sur. 
violencia psicológica, donde las regiones del norte y centro del país ${ }^{5}$ salen por arriba de dicho promedio.

A continuación se presentan algunos fragmentos que ilustran la violencia física con robo de que son objeto los migrantes deportados por la policía ya en territorio mexicano. Manuel, de 39 años, originario del Estado de México, estaba caminando como a las siete de la mañana por las vías del tren...

Iba a una fábrica donde me dijeron que contratan a gente sin papeles, y caminaba por las vías para no perderme, porque no conozco por acá (Tijuana), estaba cerca de un centro comercial que se llama el Calimax.

Ahí llegó una patrulla, se bajaron dos policías y uno de ellos me dijo que tenía un reporte de que alguien estaba incendiando esa zona, y yo era el único que caminaba por ahí a esa hora, me empezó a hacer preguntas, que adónde iba, que si usaba drogas, me preguntó muchas cosas, y yo le expliqué que adónde iba y que yo no usaba drogas.

Me pusieron con las manos en la patrulla para revisar las bolsas de mi pantalón, me sacaron mis cigarros, mi papel de baño y los tiraron, después me encontraron mis sesenta pesos y me los querían quitar, y pues es lo único que tenía y no me dejé, comencé a forcejear y me golpearon en el estómago y en el brazo, se me salió el aire y me tiraron al piso, ahí se me raspó el codo, me quitaron los sesenta pesos y me subieron a la patrulla, ahí me trajeron como una hora y media o dos horas, hasta que me llevaron al juez como a las 10:00 u 11:00 de la mañana, en la patrulla traían a otro, a ése si lo traían esposado, éste me contó que le habían quitado también todo su dinero y que si decía algo lo iban a llevar con unos mafiosos para que lo mataran.

Respecto a quién fue responsable de los actos de violencia en Estados Unidos, se puede señalar a los agentes de migración estadounidense, a la policía del condado, a los polleros o coyotes y a los asaltantes. Los primeros aparecen más representados con $60 \%$, como resultado de la participación de cada una de las regiones migratorias; sin embargo, por arriba de este porcentaje se colocan las regiones del centro y la tradicional. En segundo lugar aparece la policía del condado como responsable de la violencia contra los migrantes con $13 \%$, donde sólo aparecen las regiones tradicional ${ }^{6}$ y del norte de Méxi$\mathrm{co}^{7}$. En tercer término se encuentran los asaltantes como agentes de violencia

5 Región que se integra por los estados de Hidalgo, Morelos, Puebla, Querétaro, Distrito Federal, México y Tlaxcala.

6 Es decir los estados de Jalisco, Guanajuato, Michoacán, Zacatecas, Aguascalientes, San Luis Potosí, Colima y Nayarit.

7 Son Baja California, Coahuila, Chihuahua, Nuevo León, Sonora, Tamaulipas, Sinaloa y Baja California Sur. 
con $9 \%$ y por arriba de este dato las regiones del norte y la del centro ${ }^{8}$. En cambio, en el escenario mexicano, los principales responsables de los actos de violencia son la policía municipal mexicana, la Procuraduría de Justicia Estatal (PJE), los polleros o coyotes y los asaltantes; el 65\% corresponde a la policía municipal y el $20 \%$ a los asaltantes, entre otros, como los polleros o coyotes.

Pese a los atropellos que viven los indocumentados, como lo expone el Centro Latinoamericano y Caribeño de Demografía, en ocasiones el trabajador migratorio prefiere las condiciones de explotación en un país rico que su "empobrecida" libertad en su país de origen. Además, considera que la denuncia de su explotación ocasionaría su deportación inmediata; en otras palabras, su "rescate" significa su "captura" (Celade, 2003). Casos como éstos hacen que los derechos de los migrantes indocumentados sean vulnerados con regularidad, debido al miedo y al silencio que ellos guardan para no perder su único ingreso o trabajo que poseen para dar estabilidad económica a las familias que dejaron en sus países de origen. Esta condición acentúa el maltrato y la explotación de los migrantes, especialmente de los indocumentados.

A continuación se ofrecen algunos fragmentos de una entrevista que da cuenta de los distintos tipos de violencia, abandonos y maltratos que reciben los migrantes en su travesía en ambos lados de la frontera: David, de 35 años y originario de Cuernavaca, Morelos, contrata a un coyote para intentar pasar la frontera por Tecate, Baja California...

Éramos un grupo de 15 personas, pasamos a Estados Unidos. Por la montaña, caminamos mucho, casi un día y medio, ya cuando estábamos bien adentro de la montaña, el coyote le dijo a las cuatro personas que estaban hasta adelante con él que se pusieran a un lado y a nosotros, los once que quedábamos, que nos escondiéramos en una cueva, que él volvería en unas horas, y pues así lo hicimos, esperamos ahí, y nada que regresaba, así pasó día y medio, lo bueno es que yo llevaba unos quesos que le regalaría a mi esposa y un garrafón de agua, con eso nos mantuvimos los once que esperábamos en la cueva. Ya por la noche del domingo cinco de febrero, no teníamos ni comida ni agua, así que decidimos seguir caminando solos, el coyote ya nos había abandonado.

En el momento de cruzar la frontera, caminamos a oscuras por la montaña, había muchas piedras sueltas y me resbalé y caí como tres metros para abajo, y bueno, menos mal que choqué con una piedra grande, si no, me hubiera matado, porque había un desfiladero bien profundo, así que lo único que me pasó fue el golpe del hombro que me dolía mucho, porque se me quiso salir el brazo, pero seguimos caminando hasta que amane-

8 Refiere a los estados de Hidalgo, Morelos, Puebla, Querétaro, Distrito Federal, México y Tlaxcala. 
ció y llegamos hasta el freeway, ahí salió una patrulla fronteriza que nos detuvo y nos llevó a sus oficinas donde nos tomaron las huellas, la foto y algunas preguntas, ya como las cuatro o cinco de la mañana, nos sacaron para este lado [Tijuana, B.C.], ya era lunes, ese día intenté cruzar con otro coyote, pero esta vez por Otay, brincando la barda.

La primera barda la brinqué bien, está un poco alta pero no tuve problemas, pero la segunda barda es mucho más alta, como unos siete metros, no sé la verdad cuánto mide, cuando me dejé caer para el otro lado de esa barda caí mal, se me doblaron los pies, y pues el dolor y tan sólo ver que hay otra malla que está como de unos dos metros, ya no pude seguir, y ahí donde estaba tirado llegó la migra, que me dijo que subiera a la patrulla y me llevó otra vez a sus oficinas, donde me tomaron las huellas, la foto y las preguntas como el día anterior, ahí me tuvieron como seis horas, yo le pedí a un migra si me daba algo para el dolor, porque me dolía o si era posible que me revisara un médico, y el migra sólo me dijo: "Qué!! ¿No eres hombre?... Aguántate, que acaso yo te dije que te brincaras!!!, así que si te duele no es mi problema!!!”

De las entrevistas se deja entrever con claridad el abandono de los migrantes, las decisiones que toman de seguir caminando a pesar de no conocer la zona, y de noche para no ser observados por la patrulla fronteriza, expuestos a los accidentes y hasta a la pérdida de la vida; avanzan por las montañas y tienen travesías peligrosas. También se enfrentan a otro tipo de esfuerzos y riesgos al brincar bardas muy altas, pues no sólo tienen que saltar una, sino hasta tres bardas de diferentes alturas que llegan a medir hasta los siete metros. En las oficinas de la patrulla fronteriza les toman las huellas, las fotos, y les hacen el interrogatorio de rutina para el expediente electrónico, para luego deportar a los migrantes a Tijuana, Baja California, no sin antes amenazarlos de que si vuelven a pasar y los capturan, les van a dar una condena de 10 o 20 años en las cárceles estadounidenses.

Las condiciones en que se producen las detenciones y deportaciones de los migrantes ilegales conlleva siempre un riesgo de vulneración de sus derechos, como lo expresa las Naciones Unidas y la CEPAL (2006), y por lo general, expresan estos organismos dichos procedimientos se ajustan a las legislaciones nacionales, desatendiendo los convenios internacionales existentes sobre migración, hechos que dejan en un alto grado de vulnerabilidad a los migrantes.

En esta línea la violación de los derechos humanos se puede dar más como lo expone Valle (2009), cuando la normativa aplicable a personas extranjeras depende de la aplicación de una política securitista donde los extranjeros son vistos como una posible amenaza a la seguridad interna del país, cuyo ingreso y permanencia deben estar apegados irrestrictamente a las normas impuestas por el Estado receptor. El mismo autor expone que sea cual fuese la política 
migratoria de un determinado gobierno, ésta debe respetar al menos los contenidos esenciales de los derechos fundamentales otorgados a los ciudadanos.

\section{Fenómeno migratorio asociado al consumo, venta de droga, la privación de su libertad y maltrato}

Las causas del encarcelamiento y la deportación no sólo se debieron al consumo de drogas y/o alcohol y venta de droga, sino básicamente a las infracciones de tránsito y/o acumulación de multas. Al respecto, el trabajo de Alarcón et al. (2009) considera, con base en la información del Servicio de Inmigración y Control de Aduanas (ICE), que los migrantes deportados a México no necesariamente son criminales. Hay deportaciones por cuestiones como la inasistencia a una cita con el juez de Inmigración o por estar trabajando de manera indocumentada en una empresa donde se realizó una redada por parte de la policía de Inmigración. Además de una política mucho más dura en la frontera México-Estados Unidos, las redadas, las detenciones en casas, las órdenes de deportación y de prácticas en centros de detención se multiplicaron de forma alarmante en prácticas más represivas de inmigrantes con residencia en territorio estadounidense (Lejeune, 2010).

Según cifras del ICE, se conoce la evolución de las deportaciones en sus dos categorías por ser criminales o no. Respecto a estos últimos migrantes deportados no criminales, se observa una tasa de crecimiento de $62.3 \%$, al pasar de 115,827 a 187,878 deportados en 2005 y 2007 , respectivamente. En cuanto a los migrantes deportados criminales se registra un incremento de 8.5\%, al pasar de 89,635 a 97,278 deportados criminales en los mismos años.

El Servicio de Inmigración y Control de Aduanas deportó a 396,906 personas en 2011 , de las cuales $54.6 \%$ estaban clasificados como criminales, 19.6\% transgresores reincidentes de leyes de Inmigración; 11.6\% personas que acaban de cruzar la frontera sin papeles; $4.7 \%$ fugitivos de inmigración y 9.5\% otros extranjeros deportados. Los funcionarios del gobierno estadounidense indican que nunca antes habían logrado sacar del país a más fugitivos, criminales, convictos, personas que han cruzado recientemente la frontera y grandes violadores de las leyes de Inmigración, como durante el año 2011.

\section{Víctimas del maltrato y violencia que padecen los migrantes}

Respecto a dónde ocurrieron los actos de violencia que recibieron los migrantes, se observa que más del $82 \%$ de los entrevistados declaró que había 
sido en el territorio mexicano, y 16\%, en promedio, consideró que en el escenario estadounidense, sin embargo las regiones sur-sureste con $21.1 \%$ y la región tradicional con $17.8 \%$ presentan un promedio más alto. Los migrantes que aseguran haber sido sujetos de violencia en ambos lados de la frontera pertenecen a la región norte.

Sobre la denuncia de los hechos violentos contra los migrantes se tiene: a) a los que sí presentaron denuncia en México; $b$ ) los que sí presentaron en Estados Unidos; c) los que presentaron en ambos lados de la frontera; y $d$ ) los que no presentaron denuncia. Los resultados de la encuesta mencionada muestran que cerca de $86.7 \%$ no presentaron denuncia; cerca de $9.7 \%$ sí presentaron denuncia en México y; $3.2 \%$ los que sí presentan denuncia en los Estados Unidos.

La causa de por qué los migrantes no presentan denuncia obedece en primer lugar, con $52.3 \%$, a que según ellos no sirve de nada denunciar, esta respuesta se dio más en los migrantes procedentes de las regiones tradicional y sur-sureste de México. En segundo lugar, con 22\% no sabían que podrían hacer la denuncia, respuesta generalizada en los migrantes de cada una de las regiones migratorias pero de manera más significativa de quienes proceden de las regiones: tradicional y del centro. En tercer lugar, con $11 \%$, se encuentran quienes tenían miedo de represalias, sobre todo las personas que provienen de las regiones del norte y del centro. En cuarto lugar, con $5.1 \%$, se hallan quienes expresaron no tener dinero ni tiempo para los trámites, respuesta dada por los migrantes que proceden de la región sur-sureste de México. Para ilustrar este caso, se presenta un segmento de la entrevista realizada a José, de 41 años, originario de Comayahua, Honduras...

No recuerdo exactamente cuándo salí de Honduras, pero más o menos como a principios del mes de abril, ya hace casi dos meses. Yo soy campesino, pero ya la cosecha no deja pa' comer, menos pa' vestirse, así que lo platicamos mucho mi mujer y yo, y decidimos que pa' que nuestros hijos lo pasen mejor que nosotros, era mejor ir pa'l norte.

Así que como dos meses antes pizcamos [recolección manual de la cosecha] todo lo que la tierrita que tenemos nos dio, y lo metimos al establo, metimos maíz, pa' que no falte tortilla y pa' que venda y tenga pa' los frijolitos, y también pizcamos algo de frijol, no más pa'l consumo de la familia.

Me junté unos 2000 pesos mexicanos y me despedí de mi gente, llegué a Guatemala y de Guatemala hasta Arriaga, Chiapas, México, ahí me subí al primer tren hasta Coatzacoalcos, Veracruz, ahí cambié a otro tren, ese tren pasa por un lugar que se llama Orizaba, ahí mismo en Veracruz, pero es la pasada más dura, porque hace un frío que en ese momento uno se arrepiente de haber salido de su casa, se lo juro que imaginaba mi 
camita y mis tres cobijas, pero ya que le va uno a hacer, pues es que nos tocó montarnos en un vagón sin techo y a ésos les cae todo el sereno de la madrugada.

Nos tocó abrazarnos entre todos, conocidos y desconocidos, hombres, mujeres y hasta niños, que estos pobrecitos pensé que se morirían de tanto frío, en ese momento me provocó bajarme del tren y regresar a mi casa. Yo le aseguro que si conozco a alguien en Honduras que quiera venir, yo sí le digo que es cosa seria y de pensarlo bien, porque es duro el camino, yo ahora le voy a seguir porque ya ni modos, ya estoy a un paso del otro lado.

El jueves pasado fui a comprar una tarjeta de teléfono pa' llamarle a mi esposa, yo tranquilo caminando y que se para una patrulla, se bajaron dos policías, un hombre y una mujer, y que sin más me empujaron y me empezaron a insultar, que si de dónde era, si quería robar, si traía droga, en fin, puras cosas que yo no entendía porque me estaban diciendo eso, sin entender nada, porque fue muy rápido, me subieron a la patrulla, ahí venían muchos, veníamos ya unos encima de otros y a todos nos llevaron a la cárcel.

Cuando llegamos a un lugar que le dicen la veinte [cárcel] nos metieron a todos, pero ese lugar apestaba, había orines, mierda de gente, en fin de todo, yo hasta me vomité, como a las tres horas se acercó un policía y nos gritó: "El que quiera salir, que pague su multa”.

Pues como la verdad es que yo ni la debía ni la temía, le dije al policía que quería pasar a ver al juez, y sí, sí me pasaron a ver al juez, y que me pregunta muy grosero: "Y tú qué quieres, qué estabas haciendo", pues le dije que sólo iba a comprar una tarjeta de teléfono, que estaba en la casa del migrante y que no era delincuente, pero sólo me preguntó que de dónde era y le dije que era de Honduras, y se echó a reír y me dijo: "Pues nomás por eso son $\$ 300$ pesos de multa y para adentro 36 horas".

Los migrantes oponen resistencia al ver que las autoridades de la policía quieren despojarlos de sus pocos recursos monetarios para poder comer y trasladarse a buscar trabajo, motivo por el cual sufren violencia física: golpes en las diferentes partes del cuerpo, en particular en el estómago, lugares donde incluso pueden resultar lesiones internas, pero como no están expuestas, como los moretones, es más difícil demostrarlas. Además, son amenazados para que no se atrevan a denunciar los actos violentos de que han sido objeto por parte de las autoridades. Primero se les despoja de sus bienes y dinero, luego se les arresta y después se les amenaza con entregarlos a la delincuencia para que les quiten la vida y los desaparezcan.

En este sentido, (Foucault, 2002:15) considera que sin tocar el cuerpo, la guillotina suprime la vida, del mismo modo que la prisión quita la libertad, o una multa descuenta sus bienes para que los migrantes puedan salir de la cárcel o prisión. 
Es necesario recalcar aquí que la protección de los derechos humanos es trascendental, fomentando la inclusión e integración social de los migrantes, y así contribuir a vivir económicamente productivos y sin discriminación. De acuerdo con lo señalado por el Grupo Mundial sobre Migración (GMG) (2010), "la protección de estos derechos no es sólo una obligación legal; es, además, una cuestión de interés público que está intrínsecamente relacionado con el desarrollo humano". En este sentido, de acuerdo con las estadísticas y los relatos de los migrantes, se puede observar que los derechos son vulnerados por las autoridades tanto estadounidenses como de México, de ahí la necesidad de que los gobiernos busquen la implementación de programas y políticas migratorias que protejan a la población migrante, donde se incluyan a las autoridades y responsables de todos los niveles, para que la aplicación y el respeto al derecho sea una prioridad.

La realidad que vive hoy toda la población migrante en el mundo invita a los responsables a adoptar programas con visión universal de los derechos humanos, como base común para todos los estados, donde se respete a cada persona migrante independientemente de su origen, raza, sexo, condición socioeconómica, etcétera, y donde prevalezca el respeto a la dignidad humana sin discriminación.

Finalmente, el Informe sobre Desarrollo Humano del Programa de las Naciones Unidas para el Desarrollo (PNUD) de 2009 señala:

Es cada vez más aceptado que su proliferación [por lo que se refiere a las clasificaciones de la migración] confunde en lugar de aclarar los procesos que subyacen a la decisión de emigrar, con posibles efectos perniciosos en las decisiones de política. Si bien la movilidad humana es cada vez más global y frecuente, la distinción tradicional entre la migración voluntaria, forzada, regular o irregular, temporal, estacional o a largo plazo y permanente es cada vez menos clara. Ello se traduce a favor de un argumento mucho más convincente para abordar los derechos de todos los migrantes de manera holística, independientemente de sus razones para emigrar y de su condición jurídica, reforzando al mismo tiempo la protección que se ha creado en relación con algunos grupos específicos.

Lo anterior lleva a reflexionar a profundidad la situación de los migrantes, dadas las condiciones internacionales de violencia. Las poblaciones se ven obligadas cada día a dejar sus países de origen, y los desplazamientos forzados por la violencia, por ejemplo, es una muestra de ello. Según el Alto Comisionado de las Naciones Unidas para los Refugiados (ACNUR) (2014), hasta 2013 había 51.2 millones de desplazados forzosos en el mundo, de los cuales 33.3 millones son desplazados internos. Asimismo, se calcula que 32,200 personas son forzadas a abandonar sus hogares diariamente. 
La violencia que vive México y Honduras ha generado nuevos desplazamientos causados por la violencia creada por el crimen organizado y la militarización. En México se estima que aproximadamente 160,000 personas fueron desplazadas a nivel interno y externo, mientras que en Honduras se calculan que son 17,000. Las principales causas del desplazamiento son las amenazas, extorsiones, secuestros, pagos de uso de piso, reclutamiento forzado, censura, y persecución política y social. Muchos de estos desplazamientos han sido generados con el fin de controlar zonas de cultivo o con recursos naturales (Migrantes, 2012). Este tipo de violencia, como lo plantea el PNUD, genera una confusión en la clasificación de la migración, de ahí la urgencia de diseñar programas y políticas transversales de atención a la población migrante para no vulnerar sus derechos fundamentales, que ya de por sí han sido violentados en sus lugares de origen, como lo relata José, de 41 años, originario de Comayahua, Honduras.

\section{Reflexión final}

Del presente trabajo se desprenden las siguientes reflexiones:

Primero: A pesar de que se han incrementado las deportaciones de los inmigrantes, lo cual representa salir del país de destino, las causas se asocian a faltas administrativas de tránsito, ya sea por haber consumido alcohol y/o por las multas acumuladas de tránsito e incluso sólo por no haber asistido a una cita con el juez.

Segundo: Están los casos de los inmigrantes con residencia legal en Estados Unidos; cuando se ven involucrados en una falta administrativa, las autoridades de Inmigración les retienen los documentos que acreditan su residencia legal en el país y los castigan no permitiéndoles entrar al país hasta cumplir diez, quince o veinte años de haber sido deportados.

Tercero: En los dos escenarios - estadounidense y mexicano- los migrantes son objeto de violencia. Respecto al primero, los migrantes padecen violencia física y, en el mexicano, además de ésta, sufren el robo de sus bienes y pertenencias.

Cuarto: Los migrantes originarios de la región norte no son tan vulnerables ante el robo de sus bienes como en el caso de quienes proceden de las otras regiones migratorias, según se observa en los datos de la encuesta. Esto obedece a que la región norte del país está más familiarizada con los migrantes porque es por esta frontera donde se dan las deportaciones de Estados 
Unidos hacia México, o también porque esta frontera es la puerta de entrada de los migrantes indocumentados a Estados Unidos.

Quinto: La violencia contra los migrantes no es denunciada, pues ellos consideran que no sirve para nada, lo cual muestra la corrupción que prevalece en las autoridades mexicanas. El 80\% de los migrantes dijo haber sido víctima de violencia en el territorio mexicano sin esgrimir los hechos violentos que se ejercen. También se observa una mayor tendencia a presentar una denuncia de la violencia por parte de algunos migrantes de la región centro y con estudios universitarios.

Sexto: Es necesario proporcionar información a los migrantes vulnerables a situaciones de violencia, pues cerca de una quinta parte de ellos declaró no saber o desconocer los mecanismos para denunciar y minimizar las situaciones o exposición a la violencia contra su persona y la violación de los derechos.

Vale la pena mencionar aquí a Muñoz (2009: 11):

En la actualidad el atributo de universalidad es consubstancial al concepto de derechos humanos. Y esto ocurre en dos sentidos: Por un lado, desde el punto de vista territorial, los derechos humanos deben ejercerse, aplicarse y garantizarse en cualquier país y en todo su territorio, sin importar la forma de gobierno que adopte el Estado. Más aún, cualquier tipo de gobierno tiene como espejo para observar y determinar su nivel de legitimidad el marco jurídico-político de los derechos humanos y de toda la normatividad existente al respecto. Por otro lado, desde el punto de vista sociodemográfico, no puede existir razón alguna para que una sociedad desconozca los derechos a cualquier persona o grupo humano independientemente de su origen étnico, género, edad, condición social o estatus legal.

Lo anterior lleva a pensar que el compromiso de los gobiernos es hacer cumplir los convenios y tratados que a lo largo de la historia se han firmado con el fin de proteger a los migrantes en el mundo. Son muchos los esfuerzos que se han adelantado a nivel internacional para la protección de las poblaciones migrantes, pero aún hoy se siguen vulnerando sus derechos pese a este mundo globalizado y de libre "apertura". El reto al libre tránsito sin discriminación es la tarea de los gobiernos para lograr una verdadera apertura de las fronteras en el mundo, no sólo comercial sino humana.

\section{Agradecimientos}

Las autoras expresan un especial agradecimiento a María, Manuel, David y José, por haber compartido a través de la entrevista a profundidad su vida y 
trayectoria dentro de la experiencia migratoria que han vivido. A Stephane Vinhas de Médicos del Mundo (Francia), quien fue coordinador del proyecto en Tijuana, por haber suministrado la información necesaria para el desarrollo de este artículo. Y a las Facultades de Ciencias Sociales de la Universidad Autónoma de Zacatecas y de Economía y Relaciones Internacionales de la Universidad Autónoma de Baja California (UABC) por concedernos un espacio académico para nuestro desempeño profesional.

\section{Bibliografía}

Alarcón, Rafael et al. (2009), "La crisis financiera en Estados Unidas y su impacto en la migración mexicana”, en Revista Migraciones Internacionales, vol. 5, núm. 1, México: Colegio de la Frontera Norte.

Alto Comisionado de las Naciones Unidas para los Refugiados (ACNUR) (2014), El coste bumano de la guerra-Tendencias globales 2013. Ginebra Suiza.

Centro Latinoamericano y Caribeño de Demografía-División de Población de la CEPAL (2003), Resumen y aspectos destacados de la Conferencia hemisférica sobre migración internacional: derechos humanos y trata de personas en las Américas, Serie Seminarios y conferencias, núm. 33 (LC/L.2012-P), Santiago de Chile: Comisión Económica para América Latina y el Caribe (CEPAL), Publicación de las Naciones Unidas, núm. de venta: S.03.II.G.174.

Ceriani, Pablo et al. (2015), "Indicadores de derechos humanos para migrantes y sus familias", en Documento de Trabajo, núm. 5, Washington D.C. Global Knowledge Partnership on Migration and Development (KNOMAD).

Cortés, Fernando y Rosa Ma. Rubalcava (1987), Métodos estadísticos aplicados a la investigación en ciencias sociales: análisis de asociación, México: Colegio de México.

Foucault, Michel (2002), Vigilar y castigar: nacimiento de la prisión, Argentina: Siglo XXI.

Fuentes, Gabriela y Luis Raúl Ortiz (2012), "El migrante centroamericano de paso por México, una revisión a su condición social desde la perspectiva de los derechos humanos", en Convergencia Revista de Ciencias Sociales, vol. 19, núm. 58, México: Universidad Autónoma del Estado de México.

Cornelius, Wayne (2001), "Death at the Border: Efficacy and Unintended Consequences of U.S. Immigration Control Policy", en Population and Development Review, vol. 27, núm. 4, University of Michigan.

Gómez, Diana Andrea (2010), "Migraciones internacionales, crisis mundial, nuevas realidades, nuevas perspectivas", en Análisis Político, vol. 23, núm. 70, Bogotá: Universidad Nacional de Colombia, Universidad de Salamanca.

Gómez, Érick (2010), “Migración, desplazamientos forzados y trata de personas", en Revista Trabajo Social, núm. 19, México: Universidad Nacional Autónoma de México.

Martínez, Jorge (2003), El mapa migratorio de América Latina y el Caribe, las mujeres y el género, Proyecto Regional de Población Celade UNFPA (Fondo de Población de las Naciones Unidas), Serie Población y desarrollo 44, Santiago de Chile. 
Médicos del Mundo (2008), Encuesta sobre la situación de la salud de los migrantes y su acceso a la salud, Tijuana, Baja California. Frontera de México con Estados Unidos. Ciudades de Tijuana y Mexicali, Médicos del Mundo Francia.

Migrantes, C. I. (2012), Desplazamiento forzado y necesidades de protección generados por nuevas formas de violencia y criminalidad en Centroamérica, Centro Internacional para los Derechos Humanos de los Migrantes (CIDEHUM), Costa Rica.

Muñoz, Jairo (2009), “Migraciones, derechos humanos y ciudadanía universal. Diálogos migrantes", en Revista del Observatorio Colombo-Ecuatoriano de Migraciones OCEMI, núm. 2, Bogotá: Fundación Esperanza.

Naciones Unidas, CEPAL (2006), Migración internacional, derechos humanos y desarrollo en América Latina y el Caribe, síntesis y conclusiones", en Trigésimo primer periodo de sesiones, Montevideo, Uruguay, del 20 al 24 de marzo.

Programa de las Naciones Unidas para el Desarrollo (PNUD) (2009), Informe sobre Desarrollo Humano Superando barreras: Movilidad y desarrollo humanos, Nueva York.

Refugiados, A. C. (2014), Tendencias 2013. El coste humano de la guerra, Ginebra: Alto Comisionado de las Naciones Unidas para los Refugiados.

Valle, Alex (2009), "El derecho a tener derechos”, en Pérez, Nicole et al. [eds.], Los Derechos en la movilidad humana: del control a la protección, Ecuador: Ministro de Justicia y Derechos Humanos.

\section{Recursos electrónicos}

Bassegio, Luiz y Juan A. Plaza Gallegos (2007), México: I Cumbre de comunidades migrantes latinoamericana - Es imperativo que se escuche fuerte la voz del migrante, en Primera Cumbre de Comunidades Migrantes Latinoamericanas, México: CCMLA. Disponible en: http://www.cumbredemigrantes.org/index.php?id=119\&tx ttnews\%5Btt_news\%5D=35\&tx_ttnews\%5BbackPid\%5D=63\&cHash=dfcbaf03c7 (22 noviembre 2011).

Grupo Mundial sobre Migración (2010), Declaración del Grupo Mundial sobre Migración relativa a los derechos humanos de los migrantes en situación irregular, 30 de septiembre de 2010. Disponible en: http://www.ohchr.org/en/NewsEvents/Pages/DisplayNews. aspx ?NewsID=10396\&LangID=s. (19 de enero 2015).

Instituto Nacional de Migración (2007), Aspectos básicos de la migración en México, México: Senado de la República, Segob. Disponible en: http://www.senado.gob.mx/ comisiones/LX/parlatino/content/comisiones/9/doc2.pdf. (18 de febrero de 2013).

Lejeune, C. (2012), Los inmigrantes en los Estados Unidos: Los extranjeros ilegales en su camino de convertirse en emergentes "temas posibles". Disponible en: http://biologiacarlota1. blogspot.com/2010/04/los-inmigrantes-en-los-estados-unidos-html. (10 de diciembre de 2012).

Morales, Ignacia (2008), “Migrantes mexicanos ilegales en Estados Unidos y violación de los derechos humanos", en Iberoamérica Global, vol. 1, núm. 4, Publicación académica digital. Disponible en: http://www.iberoamericaglobal.org/\#!vol1-num4-nov2008/ clm69 (15 de marzo de 2013). 
Perelló C., María Isolda (2013), "La ayuda humanitaria y defensa de los derechos humanos de los migrantes en torno a la Frontera Norte de México", en Quaderns Electrónics sobre el Desenvolupament Humà i la Cooperació, núm. 1, Universitat de València. Disponible en: http://www.uv.es/edhc/edhc001_perello.pdf(11 de junio de 2015).

Martha Guerrero Ortiz. Doctora en Ciencias Sociales con especialidad en Estudios Regionales por el Colegio de la Frontera Norte de México. Con maestría en Ciencias Sociales y licenciatura en Economía, ambas por la Universidad Autónoma de Zacatecas. Actualmente es docente e investigadora Titular C, adscrita a la Unidad Académica de Ciencias Sociales. Pertenece al Sistema Nacional de Investigadores (SNI), Nivel 1, con perfil deseable PROMEP; integrante del Sistema Estatal para Prevenir, Atender, Sancionar y Erradicar la Violencia contra las Mujeres, en calidad de vocal, desde el año 2010. Líneas de investigación: migración internacional, familia y violencia de género. Publicaciones recientes: "Modelo de desarrollo y derechos humanos con perspectiva de género", en Revista Asuntos, núm. 22, primer semestre, Colombia: Universidad de Manizales (2012); "Migración de retorno y las consecuencias sociales, económicas y escolares en el estado de Zacatecas", en el Primer Congreso Latinoamericano de Ciencias Sociales, 2012, Zacatecas; "Emigración internacional y retorno de migrantes por regiones migratorias en México", en Memorias de la XVI Reunión Internacional La Frontera: una nueva concepción cultural (2012).

Martha Cecilia Jaramillo Cardona. Doctora en Ciencias Sociales con especialidad en Estudios Regionales por el Colegio de la Frontera Norte de México. Con maestría en Desarrollo Regional de la Pontificia Universidad Javeriana de Bogotá, Colombia; docente e investigadora de la Facultad de Economía y Relaciones Internacionales de la Universidad Autónoma de Baja California (UABC) en la ciudad de Tijuana. Pertenece al Sistema Nacional de Investigadores (SNI), Nivel 1, perfil deseable Promep, encargada de la Academia de Ciencias Sociales y Humanas de la Facultad de Economía y Relaciones Internacionales. Líneas de investigación: descentralización, salud y políticas públicas. Publicaciones recientes: "Es México, sólo cambié de frontera: de la vida rural al mundo citadino una experiencia de crecimiento personal", en Revista Trabajo y Sociedad, Argentina (2012); "Comportamiento político: un aporte a la discusión desde una perspectiva teórica y metodológica”, en International Journal of Latin American Studies. Journal of the Institute of Iberoamerican Studies, Busan University of Foreign Stu- 
dies (2012); "Risk behaviors among undocumented immigrants in Northern Mexico: 2006-2007”, en Social Medicine, New York (2011); Salud y politicas públicas en Baja California: más que un remedio, una enfermedad, México: Universidad Autónoma de Baja California (2011).

Recepción: 11 de agosto de 2014.

Aprobación: 25 de junio de 2015. 